\title{
DESCONCENTRACIÓN MUNICIPAL EN ESPAÑA: LOS CASOS COMPARADOS DE MADRID Y BARCELONA
}

\section{MUNICIPAL DECONCENTRATION IN SPAIN: THE COMPARATIVE CASES OF MADRID AND BARCELONA}

\author{
Rubén Tamboleo García \\ Universidad Complutense de Madrid. Madrid/España. \\ tamboleo@cps.ucm.es \\ https://orcid.org/0000-0003-1631-0815
}

\begin{abstract}
Recibido/Received: 16/06/2020
Modificado/Modified: 02/11/2020

Aceptado/Accepted: 08/12/2020

RESUMEN

El modelo de Gobiernos Locales de España ha sufrido de problemas propios que se han quedado alejados del debate político y también del científico y del académico. Las Ciudades de Madrid y Barcelona cuentan con un estatus especial a través de leyes especiales, y por su tamaño e importancia cuentan con problemas específicos. De ahí la importancia de comparar las 2 ciudades en su organización inframunicipal y cómo se han establecido sus distritos para la representación política, la participación ciudadana y la política pública. Para poder estudiar este fenómeno de organización administrativa en el ámbito local de España se comparan los dos modelos de desconcentración en sus distritos y cómo se organizan estos según la regulación legal. Con la comparación podemos ver como las últimas reformas son sólo en cuanto a la participación ciudadana pero no se ha reformado la organización y estructura del modelo de distritos tras cambios de población importantes.
\end{abstract}

\section{PALABRAS CLAVE}

Gobierno local, gobernanza local, distritos, participación ciudadana, democracia participativa

\section{SUMARIO}

1. Introducción. 2. Los distritos en las grandes ciudades. 3. La desconcentración en Madrid. 3.1 la reforma del consejo territorial de 2008. 4. La desconcentración en Barcelona. 5. Puntos de divergencia y de convergencia. 6. Conclusiones. 7. Bibliografía

\begin{abstract}
Spain's Local Government model has suffered from problems of its own that have been left out of the political debate and also from the scientific and academic ones. The Cities of Madrid and Barcelona have a special status through special laws, and due to their size and importance they have specific problems. Hence the importance of comparing the 2 cities in their inframunicipal organization and how their districts have been established for political representation, citizen participation and public policy. In order to study this phenomenon of administrative organization at the local level in Spain, the two models of deconcentration in their districts are compared and how these are organized according to legal regulations. With the comparison we can see how the latest reforms are only in terms of citizen participation, but the organization and structure of the district model has not been reformed after major population changes.
\end{abstract}




\section{KEYWORDS}

Local government, local governance, districts, citizen participation, participatory democracy.

\section{CONTENTS}

1. Introduction. 2. The districts in the big cities. 3. Deconcentration in Madrid. 3.1. The 2008 territorial council reform 4. Deconcentration in Barcelona. Points of divergence and convergence. Conclusions. 7. References

\section{INTRODUCCIÓN}

Como decía en los congresos científicos en los que he participado con esta línea de investigación, fruto de lo cual es el presente trabajo, cuando el actual sistema democrático español ha superado los cuarenta años de funcionamiento, y de forma habitual se pone a través de la opinión publicada alguno de sus órganos más representativos en cuestión y en constante evaluación, ha quedado pendiente y olvidada la reforma de nuestra planta de gobierno local, como una de las necesidades de racionalización administrativa, que siempre se queda en un cajón. Aproximar la política local a los ciudadanos es un reto en España, y serviría para establecer espacios de democracia deliberativa entre otras ventajas, puesto que, si ésta debiese de ser la que más les interesase a los ciudadanos, y por lo tanto la que más participación tuviese, se ha podido comprobar en uno de sus indicadores, la participación electoral, que suscita menor interés tras sucesivos procesos electorales. Así, nos preguntamos cómo se están gestionando los modelos de la participación ciudadana en las políticas locales de las dos grandes ciudades españolas, -que además gozan de un régimen especial-, y cómo se organizan sus modelos en el análisis comparado que en este artículo se trata. Los Gobiernos Locales y los espacios de participación en las grandes ciudades en España han sido estudiados por diferentes autores tales como Vallés y Brugué, 2001, Montañés Serrano, 2013, Valero López, 2013, Pineda Nebot y Pires, 2014 y Martín Baeza, 2014 entre otros. Sin embargo, el área de los espacios de participación inframunicipal y la desconcentración local en favor de los distritos de las grandes ciudades en España es el área que más campo de estudio nos deja con ámbitos ignotos que requieren del estudio de la Ciencia de la Administración, especialmente en el caso de Madrid y Barcelona, las dos grandes ciudades en España por sus magnitudes políticas, económicas, y demográficas; y que son las dos principales regiones metropolitanas en España, conservando su papel preponderante en el sistema de ciudades como así reconocen Díaz Orueta et al. (2018). Las dos han realizado su desconcentración de la de la representación política y de la gestión administrativa con dos modelos diferenciados, tras la planta municipal surgida de la Constitución de 1978 y de la Ley Reguladora de Bases del Régimen Local de 1985, con sus respectivos reglamentos municipales sometidos a la aprobación del pleno.

El campo del estudio de la desconcentración municipal nos deja mucho espacio para la investigación, en el que hay que insistir en la necesidad de diferenciar entre descentralización y desconcentración, para diferenciar entre las Unidades de Gobierno con autoridades electas, y subunidades de gobierno con autoridades delegadas entre otras temáticas como hacen autores como Martí Costa et al. (2018). Actualmente, las grandes ciudades necesitan un modelo de desconcentración lo más eficaz posible, para acercar las políticas públicas a la ciudadanía, y la posibilidad de que ésta intervenga de forma directa en aquellas temáticas que más le afecten. En España no hay un único modelo, y cada gran municipio lo ha desarrollado de manera independiente con más o menos éxito. 
La metodología fundamental de este trabajo es una de las más adecuadas en la Ciencia Política y de la Administración, la Política Comparada o la metodología comparada, trayendo para este trabajo dos casos de estudio pertinentes por sus rasgos para la comparación como Madrid y Barcelona, según criterios como los de Laiz y Román Marugán (2003). Con este marco de trabajo, se estudia un análisis comparado con los diferentes rasgos que configuran cada modelo: número de distritos, configuración de la representación política en ellos, número de plenos de distrito al año, número de vecinos por distrito, y tipos de órganos de participación vecinal, como principales características.

\section{LOS DISTRITOS EN LAS GRANDES CIUDADES}

Los distritos, divisiones territoriales dotados de órganos de gestión desconcentrada, cumplen 3 funciones fundamentales: desconcentración de la gestión, representación, y participación vecinal, y se integran en un marco en el que "adquieren un nuevo protagonismo y revitalizan su papel no sólo como prestadores de servicios públicos, sino también su papel político como organizaciones para la educación, la participación y la democracia política" (Canales Aliende, 2011:42). Entendiendo estos como una herramienta para canalizar las necesidades de las nuevas formas de gobernabilidad urbana, integrados en la personalidad jurídica única respectiva de cada ayuntamiento tal y como recogen Carrillo Barroso y Bañón i Martínez, (1997) y Brugué y Gomá, (1998). Además de que, en los grandes ayuntamientos, deberán de ser el epicentro para la modernización administrativa, la cual "incluye tres planos conectados pero independientes: la flexibilización organizativa y la agilización de las relaciones con los ciudadanos/clientes; la redefinición de la distribución territorial del poder tanto en el plano nacional como en el espacio europeo; y la adaptación a las directrices y acuerdos comunitarios" (Bañón i Martínez, 1993:9). Epicentro que podrán ser si en el conjunto de cada municipio, manejan un porcentaje significativo del presupuesto total del ayuntamiento, siendo este uno de los principales objetivos que se tendrán que plantear para que puedan ser órganos eficaces.

La Ley Reguladora de Bases de Régimen Local, se refiere a los distritos, dentro del Título $\mathrm{X}$ de Régimen de Organización de los municipios de gran población (entendidos estos por razones de población, capitalidad y razones especiales) en el artículo 128, estableciendo en el punto 1 que "los ayuntamientos deberán crear distritos, como divisiones territoriales propias, dotadas de órganos de gestión desconcentrada, para impulsar y desarrollar la participación ciudadana en la gestión de los asuntos municipales y su mejora [...]". Y mientras que nos indica en su punto 3 que "La presidencia del distrito corresponderá en todo caso a un concejal", las competencias que deberá de tener un presidente de Distrito quedan para los respectivos Reglamentos Orgánicos, por lo que se podrían entonces establecer concejales como presidentes de diferentes mayorías políticas en cada distrito, sin afectar a la gobernabilidad global del ayuntamiento, regulando de manera adecuada las respectivas competencias, siendo este el principal aspecto político del distrito que tiene en esencia, dos opciones: dar al órgano de representación política del distrito una proyección de la representación política que tiene el ayuntamiento, aplicándose ésta de manera homogénea a todos los distritos; o por el contrario, dar a cada distrito la representación electoral que ha tenido en cada uno de ellos. 


\section{LA DESCONCENTRACIÓN EN MADRID}

La ciudad de Madrid articula su desconcentración territorial en torno a 21 distritos. Las fuentes que afectan a toda su organización son además de la propia Constitución Española, la Ley 7/1985 Reguladora de Bases del Régimen Local, la Ley 22/2006 de Capitalidad y de Régimen Especial de Madrid (que en este caso redunda en lo establecido en la LRBRL), tres Reglamentos Orgánicos: el R. O. del Gobierno y de la Administración del Ayuntamiento de Madrid, el R. O. de los Distritos de la Ciudad de Madrid, el R. O. de Participación Ciudadana del Ayuntamiento de Madrid, el Reglamento de Funcionamiento de los Consejos Territoriales de los Distritos, y finalmente por el Reglamento de los Foros Locales. Mínima regulación legal que a juicio de Prieto Romero resulta "suficiente, puesto que la organización municipal en distritos, las funciones que éstos han de desarrollar, su organización interna, etc., son aspectos que entran de lleno en el ámbito de la autonomía local y de la potestad de autoorganización municipal" (Prieto Romero, 2007:191).

Para la convergencia de las políticas de los distritos, el ayuntamiento de Madrid, en el Área Delegada de Vicealcaldía, tiene el Área de Coordinación Territorial, dirigida por un concejal, bajo la cual está la Dirección General de Coordinación Territorial, que despliega dos Secretarías Generales Técnicas: la de Coordinación Territorial de Distritos y la de Planificación y Apoyo, cada una de las cuales se encarga de cuatro áreas de gestión.

Los distritos ejercen competencias en las materias locales que les hayan sido delegadas por el alcalde y, según reza el Reglamento Orgánico (en adelante R. O.) de los Distritos de la Ciudad de Madrid algunas tales como: parques y ajardinamientos, movilidad, educación, autorización de uniones civiles, cultura, seguridad, servicios sociales y otras.

Para lo cual cada distrito cuenta con la siguiente estructura: la Junta Municipal del distrito, que articula la representación política mediante las vocalías, tanto de vecinos como de concejales, con el concejal-presidente de distrito a la cabeza, nombrado y cesado por el alcalde; el vicepresidente de la junta municipal, que sustituye al concejal titular, el coordinador del distrito (antes gerente), nombrado por la Junta de Gobierno para impulsar la labor política, una figura que fue reformada en el año 2016 y que ahora se llama coordinador en vez de gerente sin que en la práctica haya tenido grandes cambios significativos, bajo las órdenes del concejal-presidente; el secretario del distrito, que trabaja por delegación del secretario del ayuntamiento; la Junta de Portavoces, integrada por los portavoces y portavoces adjuntos de cada grupo político con representación municipal que se suman a la presidencia; y la Junta de Seguridad, presidida por el concejal-presidente constituida como órgano de coordinación en materia de seguridad en el distrito en la que formalmente se sienta el Comisario de la Policía Nacional jefe de la Comisaría Nacional en el distrito y el Jefe de la Unidad de la Policía Municipal en el distrito. Siendo estos anteriores los órganos de gobierno, y como órganos de participación en Madrid encontramos dos (tres si estudiásemos las ya extinguidas Mesas de Convivencia): el Consejo Territorial del Distrito, en el cual se articula la participación de las asociaciones ubicadas en el distrito, que cada cuatro años eligen a sus asociaciones por tipologías para que las que cuenten con mayor apoyo tengan un representante en el Consejo; y el Consejo de Seguridad; instrumento de participación para la ciudadanía de forma directa y a través de asociaciones, proyección de la Junta de seguridad, y que es un Consejo creado por convenio con el Ministerio del Interior en 1997 en el que participan bajo la presidencia del concejal-presidente del distrito, el Comisario de la Policía Nacional de la Comisaría ubicada en el distrito, el Jefe de la Unidad de Policía Municipal del distrito, un representante del Área de Seguridad del Ayuntamiento, los representantes de los partidos políticos, y las asociaciones vecinales con domicilio en el 
distrito que soliciten intervención para así interpelar a los responsables de las Fuerzas de Seguridad en las políticas y actuaciones que estén llevando y vayan a llevar a cabo para mejorar los índices de criminalidad en el distrito.

Los Consejos Territoriales, radicados en los Distritos fueron los órganos de participación estrella durante los mandatos de los alcaldes Alberto Ruíz-Gallardón Jiménez y Ana María Botella Serrano (agotando el último mandato de éste), con capacidades de información, consultas, y recogida de propuestas sobre los desarrollos de política municipal, permitiendo acercarse a los mismos a los vecinos representados por sus asociaciones o de forma directa.

Su meta principal era la de que la administración y la ciudadanía del Distrito convergiesen en este espacio como centro de gobernanza con asociaciones, vecinos y autoridades municipales como principales actores de la misma, para así intervenir en las actuaciones de Distritos y especialmente de barrios, para que la voz de los residentes no se limitase a expresarse una vez cada cuatro años.

En los veintiún distritos de la ciudad existía hasta la reforma del año 2016 uno de estos Consejos bajo la Presidencia del concejal del Distrito, y su eliminación avanzaba que cada lado de la representación política tiene su propio modelo de participación, por lo que el estudio de las figuras respectivas nos ayuda a entender mejor esta problemática. $\mathrm{Su}$ funcionamiento quedaba recogido en el Reglamento de Participación Ciudadana.

En los veintiún distritos existía un Consejo Territorial hasta el año 2017 bajo la dirección del concejal del distrito, y con convocatoria ordinaria cada tres meses. Su regulación estaba recogida en el Reglamento de Participación Ciudadana del Ayuntamiento. Todo esto cobra importancia actual porque la nueva reforma prevista para el año 2021 se base en este diseño anterior con actualizaciones y modificaciones.

Según recogía el artículo 55 del R. O. de Participación Ciudadana de la Ciudad de Madrid, las tres procedencias o tipologías básicas de miembros del Consejo Territorial de Distrito son: los vocales vecinos representantes de grupos políticos (máximo 8), Los vecinos elegidos de forma aleatoria a través del Censo Electoral del Distrito (4), los representantes de entidades ciudadanas (máximo de 24). Después de esto cabe decir que los Consejos Territoriales se reunían de forma ordinaria una vez cada tres meses, pero que siguen funcionando en forma de Comisiones Permanentes, y serán estas comisiones las que eleven su trabajo al plenario del Consejo. Lo usual es que estas comisiones fueran presididas por un miembro político, es decir un vocal vecino, pues bien, para la presente legislatura en el Distrito de Tetuán, se ha optado por que sean presididas por un vecino representante de alguna de las asociaciones del Distrito, lo que supone según palabras de la Concejala Presidenta que ha tomado la decisión, y delegado la dirección en las asociaciones vecinales, "un paso más en la participación ciudadana".

\subsection{LA REFORMA DEL CONSEJO TERRITORIAL DE 2008}

Tras cuatro años desde su puesta en marcha, y ante la renovación que supusieron las elecciones de 31 de marzo de 2008, en el sector de representación de las entidades ciudadanas, el Pleno del Ayuntamiento aprobó reformas significativas para mejorar el modelo de participación ciudadana.

Se incluía en el reformado R. O. de Participación Ciudadana en su artículo 17 lo siguiente: "El Consejo Territorial podrá aprobar en cada sesión ordinaria la elevación a la Junta Municipal del Distrito como máximo tres proposiciones, las cuales se incluirán sucesivamente en el orden del día de esta a razón de una por sesión, a cuyo efecto el Consejo Territorial establecerá el orden de prioridad de las mismas". Lo cual se decidía entre el o la 
concejal respectivo y el o la vicepresidente del consejo a la sazón representante de una de las asociaciones del distrito, ocurriendo esto de forma desigual entre los 21 de la ciudad. También se producía una restricción efectiva para que los asuntos que se trataban fuesen de competencia pertinente del Distrito respectivo, o en un enfoque más flexible, que por lo menos fuesen competencia del Ayuntamiento, para así dejar para otros espacios de debate asuntos de política regional, nacional o de otros ámbitos.

Otros artículos más son reformados en el R. O. de Participación Ciudadana, que afectan sobre todo a las entidades ciudadanas, y significativa es la reforma del artículo 55, que ampliaba a 51 el número máximo de vocales del Consejo Territorial (antes el máximo era de 40), dando nuevas plazas representantes de la infancia y la adolescencia y de los Centros Municipales de Mayores y ampliando representantes de las entidades ciudadanas según la tipología de la representación de ellas.

Y se modifica también a finales de 2008 para que fuese efectivo en la práctica tras las elecciones citadas antes de 31 de marzo el R. O. de Funcionamiento de los Consejos Territoriales. En primer lugar, para adaptar su texto a las reformas del R. O. de Participación, para hacer que el Secretario del Consejo sea el Secretario del Distrito, para darle un suplente al Portavoz del Consejo, las formas de su convocatoria, reflejando en el texto reformado el uso de las nuevas tecnologías para ello, y se modifica la regulación de las comisiones del Consejo con la limitación a cuatro el número máximo de comisiones permanentes.

Además, el reglamento fijaba que las comisiones del Consejo Territorial "estarán integradas por un presidente y, además, por un máximo de 12 vocales del Consejo, que manifiesten querer pertenecer a la misma y comprometerse a participar en sus trabajos. El presidente de las Comisiones será el presidente del Consejo o persona en quien delegue. Al menos la mitad de las Presidencias serán delegadas en vocales del Consejo no pertenecientes a los Grupos Políticos municipales", esto último para dar más importancia a la representación de las entidades ciudadanas, sin recibir ninguna remuneración por ello.

La importancia del estudio del Consejo Territorial radica en que fue el modelo del Partido Popular en la ciudad de Madrid, inclinado hacia el centro derecha, y como decíamos es base para el modelo que se está pergeñando actualmente, puesto que el cambio de color político en el Ayuntamiento de Madrid para el mandato 2015-2019 hizo que se pusieran en marcha nuevos mecanismos de democracia deliberativa y de presupuestos participativos. Se establecieron los denominados como "Foros Locales" en cada Distrito con una perspectiva de autogestión y con gran influencia de las asociaciones radicadas en cada Distrito. Las áreas en las que podían trabajar no estaban delimitadas y lo que se solicitaba para su constitución es que tuviesen un mínimo de 5 integrantes con relación con el Distrito. Contaban con el apoyo de un Dinamizador. Además, se constituyó la Plataforma "Madrid Decide" con la que se reservaba una pequeña parte del presupuesto público para el desarrollo de iniciativas propuestas por la ciudadanía. A través de la plataforma se anunciaban una serie de fases como lanzamiento de propuestas, estudio de la viabilidad, recogida de apoyos, votación y resultados finales. En el lanzamiento de propuestas se recogían pidiendo que tuvieran especial incidencia para el escenario local, por lo que esto era el límite (no se podían proponer asuntos de los gobiernos regional, nacional o supranacional); en el estudio de viabilidad se miraba todas aquellas que por ejemplo se solapaban con otras propuestas, con proyectos ya en marcha, o que resultaban imposibles de realizar además de aquellas que se copiaban de otras ciudades o distritos y cuya necesidad o impacto sería muy distinto; en la recogida de apoyos todas las propuestas que pasaban el filtro se colgaban en la página web para que los usuarios registrados del Distrito pudieran apoyarlas o no; y en la fase de votación se votaban las más importantes para que se pudieran poner en marcha o se podía 
votar entre proyectos alternativos para por ejemplo la reforma de un espacio público como una plaza. Para todo esto se aprobó una última reforma del R. O. de Participación Ciudadana con una modificación parcial, aprobada por el Pleno del Ayuntamiento de Madrid el 24 de abril de 2018 tras pasar por las fases de consulta pública y de aprobación en la Junta de Gobierno de la Ciudad.

Con el nuevo cambio de color político con un Gobierno de coalición local desde el año 2019, se espera un nuevo modelo de incentivación de la participación en el que desaparecerán los conocidos como dinamizadores locales y serán sustituidos por técnicos de participación, todo ello con la base del Consejo Territorial aquí estudiado. Se prevé para inicios de 2021 (dependiendo de la evolución de la pandemia puesto que el Reglamento requiere que se haga de forma presencial) constituir las nuevas mesas de participación local, que se llamarán a partir de ahora consejos de proximidad, en cada Distrito de los 21 que funcionarán de forma sectorial por las áreas delegadas de Gobierno, y en las que tendrán más peso los Vocales Vecinos (figura que se asemeja a la de un Concejal, pero en el nivel inframunicipal de los Distritos y que no son electos de forma directa por la ciudadanía sino que son designados) de los partidos que ostentan las funciones de Gobierno, los cuales presidirán las Mesas Locales de participación, y que se coordinarán con los técnicos del Ayuntamiento para estudiar la viabilidad de las propuestas y que estas puedan ser o no sometidas al Pleno del Distrito y en su caso aprobadas.

También existen consejos a nivel de la ciudad para articular la participación ciudadana. Son en su mayoría consejos sectoriales por áreas de política pública o dirigidos a sectores como la juventud que articulan sus propuestas en sesiones convocadas al efecto. En la actualidad existen 10 Consejos Sectoriales constituidos en el Ayuntamient: de las Mujeres, de Consumo, de la Juventud, de Voluntariado, Foro de Diálogo y Convivencia, de la Discapacidad, de Mayores, de Asociaciones, de Cooperación al Desarrollo y de Comercio.

\section{LA DESCONCENTRACIÓN EN BARCELONA}

Las fuentes que afectan a toda su organización son además de la propia constitución, la Ley 7/1985 Reguladora de Bases del Régimen Local, y la Carta Municipal de Barcelona, además de los diferentes Reglamentos y sus reformas que ha habido.

En primer lugar, hay que destacar que Barcelona tiene 10 Distritos desde el año 1984 (11 menos que Madrid), esto se explica por qué Barcelona no realizó la anexión de municipios del área metropolitana, que sí que fue realizando Madrid, desde el 5 de junio de 1948 con Chamartín de la Rosa, hasta el 37 de julio de 1954 con Villaverde (en 6 años anexionó 12 municipios limítrofes).

En Madrid los Distritos se gobiernan como hemos visto por Juntas municipales con un Concejal Presidente a la cabeza, y en Barcelona se llaman Consejos de Distrito (órgano de participación y representación vecinal que se configura con el presidente del consejo, un número de consejeros proporcional al número de vecinos en el distrito según tramos de población, el edil de distrito y los ediles asignados, el cual se convoca de forma habitual bimensualmente) con un Concejal Presidente (que ejerce la dirección de los órganos colegiados de administración), y luego además está un Concejal de Distrito (los cuales actúan por delegación del primer edil, en las áreas de política pública que se hayan fijado en el decreto de constitución tras las elecciones municipales para los distritos siendo la autoridad de la demarcación, y dirigiendo la administración y el personal, coordina la relación entre ayuntamiento central y los órganos desconcentrados, ejecuta los acuerdos de 
los órganos colegiados y espolea los contactos del ayuntamiento con el movimiento vecinal como principales atribuciones), siendo esta la diferencia fundamental de dos tipos de Concejales con respecto a la ciudad de Madrid, además de tener también únicamente en Barcelona la Comisión de Gobierno de Distrito, que es el órgano de asistencia al edil, formado por el concejal y cinco consejeros, aunque también de forma menos estructurada en Madrid, los concejales de los distritos cuentan con dos asesores cada uno con dedicación exclusiva.

Y además de los órganos de gobierno, los órganos de participación, siendo el más importante hasta el mandato 2011-2015 el Consejo Ciudadano de Distrito (equivalente al Consejo Territorial madrileño), el cual es el espacio de consulta y de participación, integrado este por representantes de asociaciones ubicadas en el distrito, de consejos sectoriales de participación y por ciudadanos personalmente; además son órganos de participación la audiencia pública del estado del distrito, las comisiones de trabajo, los consejos de equipamiento y los consejos sectoriales.

En el Reglamento de Participación Ciudadana destaca el Consejo de la Ciudad además de regular el mecanismo de las Consultas Ciudadanas como forma de democracia deliberativa y que tuvieron su máximo exponente en el mandato 2007-2011 pero que después decayeron porque de forma usual los resultados eran contrarios a los objetivos del Gobierno Local como ocurrió con la Consulta sobre la reforma de la Diagonal (una de las avenidas más representativas de la Ciudad) en la que la propuesta del Alcalde Hereu salió ampliamente derrotada (Redacción, 2010). El Consejo de la Ciudad queda integrado por los siguientes miembros: el o la Alcalde que ocupa la presidencia la cual puede ser delegada en otro concejal, un o una concejal de cada uno de los Grupos Municipales presentes en el Ayuntamiento, un representante de cada uno de los Consejos Ciudadanos de Distrito, un representante de cada órgano de participación de la ciudad, hasta veinticinco representantes de las instituciones más representativas de la ciudad, hasta quince representantes de las asociaciones, hasta quince personas de renombre ciudadano, hasta veintiún ciudadanos elegidos por sorteo elegidos entre el padrón y el registro de participación, el Comisionado en materia de participación ciudadana y el Defensor de Agravios de la Ciudad.

Para el mandato 2015-2019 se produjo la reforma del Reglamento de Participación Ciudadana que trajo el Consejo de Barrio para cada Distrito con los consejos de barrio como órganos de participación territorial en todas las cuestiones referentes al ámbito de su demarcación. Se constituyen con el objetivo de ser un canal de participación ciudadana en el desarrollo de políticas públicas de proximidad y convivencia además de para poder avanzar en la cohesión social y mejorar la calidad de vida urbana de cada uno de los barrios de la ciudad de Barcelona.

\section{PUNTOS DE DIVERGENCIA Y DE CONVERGENCIA}

El primer punto común para la desconcentración en estas dos grandes ciudades, es que para su articulación jurídica tienen que remitirse a lo establecido en la Disposición Adicional Sexta de la Ley de Bases de Régimen Local.

En Barcelona los vocales vecinos se llaman Consejeros Municipales de Distrito, en un modelo claramente distinto, puesto que, en Madrid, garantizando la presencia de todos los grupos municipales presentes en el Pleno del Ayuntamiento, se proyecta la composición municipal según los resultados globales de la ciudad a cada Junta de Distrito, en la misma proporción y siempre el mismo número total de representantes, 29, sin atender a la población 
del Distrito. En Barcelona se opta por otro modelo, y la representación en cada distrito se hace según los resultados electorales que los partidos políticos han obtenido en el correspondiente distrito, teniendo también en cuenta la población del Distrito para establecer el número de Consejeros Municipales (en L'Eixample hay 23 Consejeros Municipales y en Les Corts 15, como dos ejemplos, el número completo de consejeros en cada Consejo de Distrito se puede ver en la figura siguiente número 1).

Figura 1: distribución de consejeros de distrito según habitantes en cada distrito de Barcelona.

\begin{tabular}{|c|c|c|}
\hline Distrito & Habitantes & Consejeros de Distrito \\
\hline Ciutat Vella & 111.290 & 23 \\
\hline Eixample & 262.485 & 19 \\
\hline Sants-Montjuïc & 177.636 & 15 \\
\hline Les Corts & 82.588 & 19 \\
\hline Sarriá-Sant Gervasi & 140.461 & 17 \\
\hline Gràcia & 120.087 & 19 \\
\hline Horta-Guinardó & 169.920 & 19 \\
\hline Nou Barris & 164.981 & 21 \\
\hline Sant Andreu & 142.598 & 19 \\
\hline Sant Martí & 221.029 & \\
\hline
\end{tabular}

Fuente: elaboración propia y datos del Instituto Nacional de Estadística.

Además, el modelo barcelonés que se ha desarrollado hasta la fecha promueve que el Concejal Presidente de cada Distritito no tenga por qué ser de un partido en el gobierno si no atendiendo también a los resultados en el Distrito, (recordemos que las competencias ejecutivas serán del concejal del Distrito que sí que será concejal del equipo de Gobierno). Por lo tanto, con estas dos figuras, Concejal de Distrito y Concejal Presidente del Consejo del Distrito, se logra un modelo más participativo.

Esto ocurre por ejemplo en el Distrito del Eixample, donde CiU ganó las elecciones municipales de 2007 con el 32’3 por ciento de los votos, y así el Concejal Presidente es de este partido para el mandato 2007-2011 fue el Ilmo. Sr. Gerard Ardanuy Mata. Y en el Distrito 4, Les Corts, el Concejal Presidente es representante de la tercera fuerza política en el Distrito, el PP con el 19'9 por ciento de los votos, y el Consejo tiene mayoría conservadora con 10 representantes de PP y CiU y luego 1 de ERC y 5 del bipartito de gobierno formado por PSC y ICV-Eu i A. Vemos como se atienen a claros criterios de proporcionalidad, según los resultados y la población del Distrito, al contrario que en Madrid, donde como decía antes se proyecta la imagen del Pleno de Ayuntamiento a cada Distrito, y con esto vemos como el modelo de Gobernabilidad de Barcelona se aproxima en cada Distrito mucho más a la realidad, gracias a aplicar el principio de que la composición de los Consejos de Distritos dependa de los resultados electorales en el territorio correspondiente.

Con esto 4 de los 10 Consejos Municipales del Distrito fueron presididos en ese mandato por concejales de la oposición, dos por concejales de CiU, uno del PP y otro de ERC. Y los otros seis del bipartito de gobierno. En Madrid el Concejal de Distrito es Concejal Presidente 
porque ejerce las facultades ejecutivas del gobierno en el Distrito y Preside la Junta Municipal, y los 21 concejales son del partido en el gobierno el Partido Popular, con una composición idéntica en cada Junta de Distrito, cuando las diferencias de población son si cogemos los dos extremos de más de 200.000 habitantes como podemos ver en la siguiente figura número 2.

En el caso de Barcelona, la composición básica se ha mantenido igual en los últimos tres mandatos 2011-2015, 2015-2019 y 2019-2021 pese al cambio de Gobierno municipal en el año 2015, mientras que en Madrid hemos observado como el cambio de color político en el gobierno ha traído consigo su propio modelo de participación, con el cambio en los vocales totales como se puede ver en la Figura 2 y con la introducción de los Foros Locales.

Figura 2: número de vocales vecinos y habitantes por cada distrito de la ciudad de Madrid.

\begin{tabular}{|c|c|c|c|c|}
\hline Distrito & Habitantes & $\begin{array}{c}\text { Vocales } \\
2011-2015\end{array}$ & $\begin{array}{r}\text { Vocales } \\
2015-2019\end{array}$ & $\begin{array}{l}\text { Vocales } \\
2019 \text { - } \\
2023\end{array}$ \\
\hline Centro & 149.718 & 29 & 25 & 30 \\
\hline Arganzuela & 146.833 & 29 & 25 & 30 \\
\hline Retiro & 125.978 & 29 & 25 & 30 \\
\hline Salamanca & 147.707 & 29 & 25 & 30 \\
\hline Chamartín & 142.626 & 29 & 25 & 30 \\
\hline Tetuán & 155.649 & 29 & 25 & 30 \\
\hline Chamberí & 145.593 & 29 & 25 & 30 \\
\hline $\begin{array}{l}\text { Fuencarral-El } \\
\text { Pardo }\end{array}$ & 220.085 & 29 & 25 & 30 \\
\hline $\begin{array}{l}\text { Moncloa- } \\
\text { Aravaca }\end{array}$ & 116.531 & 29 & 25 & 30 \\
\hline Latina & 256.644 & 29 & 25 & 30 \\
\hline Carabanchel & 253.678 & 29 & 25 & 30 \\
\hline Usera & 142.415 & 29 & 25 & 30 \\
\hline $\begin{array}{l}\text { Puente de } \\
\text { Vallecas }\end{array}$ & 244.151 & 29 & 25 & 30 \\
\hline Moratalaz & 104.923 & 29 & 25 & 30 \\
\hline Ciudad Lineal & 228.171 & 29 & 25 & 30 \\
\hline Hortaleza & 161.661 & 29 & 25 & 30 \\
\hline Villaverde & 126.802 & 29 & 25 & 30 \\
\hline Villa de Vallecas & 73.281 & 29 & 25 & 30 \\
\hline Vicálvaro & 66.439 & 29 & 25 & 30 \\
\hline San Blas & 157.367 & 29 & 25 & 30 \\
\hline Barajas & 43.423 & 29 & 25 & 30 \\
\hline
\end{tabular}

Fuente: elaboración propia y datos del Instituto Nacional de Estadística y del

Ayuntamiento de Madrid. 
Con el cambio de color político en el mandato 2015-2019 todos los Distritos de Madrid fueron igualmente para el Grupo Municipal que ostentaba la Alcaldía de Ahora Madrid. Y para el mandato 2019-2023 se han repartido de forma proporcional entre los dos Grupos Municipales del equipo de Gobierno de Partido Popular y Ciudadanos. Las competencias de los Distritos en ambas ciudades son muy similares. Y el número de plenos al año del órgano de representación político-vecinal es superior en Madrid, donde la media es de 13, y en Barcelona la media es de 7.

\section{CONCLUSIONES}

El modelo madrileño tiene una gran posibilidad para una reforma de su desconcentración que le haga más eficiente, aplicando un modelo racional de política pública, primero porque habría que adecuarlo en dos niveles, algunos barrios deberían de cambiar de distrito, y otros distritos dividirse, como es el claro ejemplo de Moncloa-Aravaca, para que Moncloa y Aravaca fuesen dos distritos diferenciados, y más acuciante es aún el caso de Fuencarral-El Pardo, donde claramente el que ahora es el Distrito más grande de la ciudad de Madrid debería de dividirse para dar lugar a Fuencarral por un lado y El Pardo por otro, no tiene sentido ninguno que los vecinos de El Pardo tengan como referencia una Junta Municipal que se encuentra a 10'8 kilómetros de su núcleo poblacional. Esto es un problema derivado de las anexiones de municipios a Madrid de 1953. El nuevo desarrollo urbano, más acuciante aún si tenemos en cuenta los conocidos como PAUs, Proyectos de Actuación Urbanística en la periferia de la ciudad que cambiaron de forma significativa la población residente en los Distritos con un proceso de desconcentración residencial que actúa como un mecanismo que, condicionado por las dinámicas de diversa escala territorial que actúan sobre la ciudad, da lugar en esta etapa a un nuevo modelo de fragmentación socio espacial de los espacios residenciales metropolitanos (Durá, 1997, p. 113) que afectan al desarrollo de las políticas públicas en todas sus vertientes, por lo que deberíamos tener en cuenta los procesos de desconcentración urbana (Mallarach Isern y Vilagrasa Ibarz, 2002) para la estructura organizativa de estas dos ciudades, lo cual impactará en su Gobernanza acorde a como se presten los diferentes servicios como recogen Bañón i Martínez y Tamboleo García en 2015.

Además debería de haber más racionalidad dentro de los servicios que ofrecen los propios Distritos: tras el estudio de caso del Distrito de Tetuán, que por ejemplo, cuenta con su por ahora único Punto de Juventud, pues bien, ese punto está justamente en el límite sur del Distrito, haciendo que los jóvenes del Distrito más alejados para llegar a pie y en autobús tengan que recorrer 4'7 kilómetros, configurándose este servicio de tal forma que beneficia tanto o más al vecino Distrito de Chamberí, y recordemos que ante quejas o sugerencias tendrán que referirse siempre a su Junta de Distrito, por esto se hace imprescindible o un plan estratégico para la situación de los servicios, o una redefinición del mapa político y administrativo de los distritos en la Ciudad.

Estos efectos hacen que el tejido social, con sus entidades ciudadanas a la cabeza, se aleje de sus administraciones e incluso se sienta perjudicado, puesto que, si tiene estas dificultades en los servicios administrativos y sociales que le ofrece su administración más cercana, el modelo de gobernabilidad y su gestión pública no avanza si no que retrocede, haciéndose necesario un plan estratégico de gobernabilidad en la ciudad de Madrid.

Otra posibilidad, -con posibilidad de implementar a través de un convenio-, sería incorporar representación de la Comunidad de Madrid, por lo menos en los nuevos consejos de proximidad, e incluso en la Junta Municipal, teniendo en cuenta que el área metropolitana 
de Madrid es gestionada por la Comunidad, y sobre todo atendiendo a que numerosas peticiones vecinales cursadas como iniciativas en los Consejos, son de competencias de la Comunidad, y por ello al cabo del año son numerosas las iniciativas que acaban siendo del tenor literal de "instar al órgano competente en...", por esto, para hacer más eficaz la gobernabilidad y la gestión de la ciudad, habría que mejorar la representatividad institucional por lo menos en el Consejo, puesto que actualmente se limita al Ayuntamiento. En el apartado de la reforma normativa del Consejo Territorial de 2008, se aumentó la representatividad de las entidades ciudadanas (número máximo de miembros de 40 a 51), pero en cuanto a incluir representantes de otras instituciones, se obvió por completo, y esto es posible, porque en la Junta Local de Seguridad, hay representantes del Ayuntamiento (el Jefe de la Unidad de Policía local en el Distrito), el Comisario Nacional de la Comisaría del Distrito, y un representante de la Delegación del Gobierno en Madrid; en esta Junta Local de Seguridad en los Distritos tenemos un modelo a seguir, que se podría implantar en el Consejo Territorial e incluso en la Junta Municipal, para así hacer más eficaz como decía anteriormente la Gobernanza en la ciudad de Madrid.

También hemos hablado de las significativas ausencias del sector de representantes de los vecinos elegidos aleatoriamente en el censo del respectivo distrito. Algunos portavoces de los consejos territoriales planteaban en su momento, que además de los vecinos o en lugar de los elegidos aleatoriamente, podría haber un sector de vecinos designados o que se pudiesen presentar a las elecciones, al igual que ya se presentan las entidades ciudadanas para mandar a sus representantes, propuesta que hacía para que esos vocales pudiesen, trabajar, más que en el plenario, en las comisiones. Es una propuesta que, en un futuro, con estos problemas de participación, debería de tenerse en cuenta, para así no despreciar a aquellos que tienen ganas de participar en las políticas públicas de su ciudad, siendo gente que colabora con los representantes de las entidades ciudadanas, pero que actualmente no tienen posibilidad de ser parte de esos órganos, porque quieren, y no pueden, y aquellos que pueden gracias al azar, no quieren, por lo que este tipo de colaboración se puede plantear en próximas reformas.

Sin embargo, considerando que el modelo barcelonés es más eficaz que el madrileño, las mejoras consideradas anteriormente serían de carácter secundario, puesto que la principal reforma sería la de la representación política; no tiene sentido que el distrito menos poblado, Barajas, y el más poblado, Latina, tengan por ahora el mismo número de representantes en su Junta Municipal, por lo que cabría aquí una reforma para establecer que el número de miembros tenga alguna proporción con la población, y que así las Juntas puedan ser más eficaces. Una forma de hacerlo sería que las Juntas de distrito con una población de hasta 100.000 habitantes tengan 15 vocales, que las mayores aumenten un vocal en razón de un vocal cada 10.000 habitantes más, redondeado la cifra al alza, y sumando uno más en caso de que el resultado fuese par, y que a partir de 250.000 habitantes no se superase el número de 30 , quedando con esta propuesta y con los datos de población del año de 2005 de la manera que muestra la siguiente tabla, que incluye la relación de vocales por habitantes en el distrito correspondiente tal y como se puede ver en la Figura 3: 
Figura 3: nueva distribución de Vocales Vecinos en cada Distrito según número de habitantes.

\begin{tabular}{|c|c|c|c|}
\hline Distrito & Habitantes & Vocales & Relación \\
\hline Centro & 149.718 & 21 & 7486 \\
\hline Arganzuela & 146.833 & 21 & 7342 \\
\hline Retiro & 125.978 & 19 & 6999 \\
\hline Salamanca & 147.707 & 21 & 7385 \\
\hline Chamartín & 142.626 & 21 & 7131 \\
\hline Tetuán & 155.649 & 21 & 7412 \\
\hline Chamberí & 145.593 & 21 & 7280 \\
\hline $\begin{array}{l}\text { Fuencarral-El } \\
\text { Pardo }\end{array}$ & 220.085 & 29 & 7860 \\
\hline Moncloa-Aravaca & 116.531 & 17 & 6855 \\
\hline Latina & 256.644 & 31 & 8279 \\
\hline Carabanchel & 253.678 & 31 & 8183 \\
\hline Usera & 142.415 & 21 & 7121 \\
\hline $\begin{array}{l}\text { Puente de } \\
\text { Vallecas }\end{array}$ & 244.151 & 31 & 8138 \\
\hline Moratalaz & 104.923 & 17 & 6558 \\
\hline Ciudad Lineal & 228.171 & 29 & 8149 \\
\hline Hortaleza & 161.661 & 23 & 7348 \\
\hline Villaverde & 126.802 & 19 & 7045 \\
\hline Villa de Vallecas & 73.281 & 15 & 4885 \\
\hline Vicálvaro & 66.439 & 15 & 4429 \\
\hline San Blas & 157.367 & 21 & 7494 \\
\hline Barajas & 43.423 & 15 & 2895 \\
\hline
\end{tabular}

Fuente: elaboración propia y datos del Instituto Nacional de Estadística.

Además, quedaría pendiente conseguir el objetivo consignado en el R. O. de Distritos de la Ciudad de Madrid de gasto desconcentrado, y es que para el mandato 2003-2007 se puso el objetivo de que los distritos gestionasen como mínimo el 13 por ciento del presupuesto municipal, para que así cada distrito pueda responder de forma propia a las demandas de sus vecinos que plantean en sus órganos de participación. No se cumplió para ese mandato, y tampoco se ha cumplido para el mandato 2007-2011, en el que además se produjo un retroceso en el porcentaje de gasto desconcentrado, alejándose aún más del objetivo consagrado en el R. O., después se estancó y para el mandato actual 2019-2023 tampoco se prevé llegar a ese antiguo objetivo. Por el contrario, en Barcelona el objetivo es superior, estando en el 15 por ciento para el gasto desconcentrado, siendo en realidad bastante alto en comparación con los otros grandes municipios españoles, y habiéndose alcanzado de manera efectiva en varios ejercicios económicos, aunque en cualquier caso, como recomendación general de gasto desconcentrado global en los distritos, podría recomendarse uno superior al 15 por ciento que quedaría por determinar (el que fuese más eficiente); el candidato del Partido Socialista Obrero Español a la Alcaldía de Madrid en las pasadas elecciones del 22 de mayo de 2011, propuso un objetivo de gasto desconcentrado global del 20 por ciento.

Para la ciudad de Barcelona hubo una propuesta de reforma, apoyada por las diferentes fuerzas políticas, aún más ambiciosa es la elección directa de los consejeros de distrito. "Ésta es una vieja aspiración de las fuerzas políticas con implantación o con representación en el 
Ayuntamiento de Barcelona, que la consideran un factor clave para incrementar la implicación de la ciudadanía en los asuntos públicos y para consolidar la notable tradición y voluntad participativa de esta ciudad" (Jiménez y Mallo, 2007:302). Es una propuesta que ya tiene un recorrido y que, aunque ha intentado rescatarse varias veces hasta ahora nunca se ha podido realizar.

Con todo ello, tenemos un marco en el que la gestión desconcentrada en las dos grandes ciudades con régimen especial de España, han conseguido logros significativos, pero a las que sin embargo les queda camino por recorrer mejorando los presupuestos de participación para hacerlos más representativos y más receptivos a las demandas vecinales, logrando así una mejor gobernanza local en cada capital, además de que se podría consensuar un modelo adecuado que asumieran todas las fuerzas políticas, puesto que como se ha resaltado en el artículo, la tendencia ideológica de cada una de ellas cuando ha alcanzado el gobierno local, ha impuesto su modelo de participación, sin modificar el modelo de organización de la ciudad en ninguno de los dos casos desde el año 1987. Así, para posteriores trabajos en esta línea de investigación, cabe examinar nuevas reformas como la que se espera con el gobierno de coalición de Madrid del presente año, con un nuevo modelo de Consejos de Proximidad para empezar a andar en el año 2021 si el Pleno municipal da el visto bueno al nuevo Reglamento. Y especialmente hay que dar cabida a trabajos críticos con los resultados y los mecanismos de la participación como hace Ruano de la Fuente en 2010, para mejorar en esta materia.

\section{BIBLIOGRAFÍA}

Bañón i Martínez, R. (1993) "La modernización de la Administración Pública española: balance y perspectivas", Política y Sociedad 13: 9-20.

Bañón i Martínez, R. y Tamboleo García, R. (2015) Ideas para la Gobernanza. Madrid: Editorial Fragua.

Brizubaela Castillo, L., (2007) "La organización de los distritos en Madrid”. En Prieto Romero, C. y Galán Galán, A., (dirs). Los distritos: gobierno de proximidad. Madrid: Thomson Civitas.

Brugué, Q. y Gomá, R., (coords), (1998) Gobiernos locales y políticas públicas: bienestar social, promoción económica y territorio. Barcelona: Ariel.

Canales Aliende, J. M., (2001) “Gobernabilidad y gestión pública”. En Olías de Lima Gete, B., (dir.). La nueva gestión pública. Madrid: Prentice Hall.

Carrillo Barroso, E., y Bañón i Martínez, R., (1997) La nueva administración pública. Madrid: Alianza Editorial.

Corrales Guillén, J. C., (2007) "La desconcentración como principio jurídico y técnica de organización”. En Prieto Romero, C. y Galán Galán, A., (dirs), Los distritos: gobierno de proximidad. Madrid: Thomson Civitas.

Díaz-Orueta, Fernando, Lourés, María Luisa, \& Pradel-Miquel, Marc. (2018) Transformando los modelos de crecimiento y cohesión: cambios en la gobernanza de Barcelona y Madrid. EURE (Santiago), 44(131), pp. 173-192. DOI: https://dx.doi.org/10.4067/S0250-71612018000100173

Durà, A. (1997) Desconcentración residencial y nueva fragmentación socioespacial en la regió de Barcelona: la diáspora de Santa Coloma de Gramanet. en Anales de geografía de la Universidad Complutense (Vol. 17, pp. 111-129).

Galán Galán, A, (2001a) La potestad normativa autónoma local.

Galán Galán, A, (2001b) La carta municipal de Barcelona y el ordenamiento local: el régimen especial del Municipio de Barcelona. Barcelona: Ayuntamiento de Barcelona.

Galán Galán, A, (2004) El R. O. local. Madrid: Ayuntamiento de Madrid. 
Jiménez Asensio, R. y Mallo Gómez, M., (2007) “La organización territorial en Distritos del Municipio de Barcelona” En Prieto Romero, C. y Galán Galán, A., (dirs), Los distritos: gobierno de proximidad. Madrid: Thomson Civitas.

Laiz, C. y Román Marugán, P. (2013) Política Comparada. Madrid: McGraw Hill.

Ley 7/1985. Ley Reguladora de las Bases del Régimen Local. Boletín Oficial del Estado, nº 80, 1985, 3 de abril, accesible en: https://www.boe.es/buscar/act.php?id=BOE-A-1985-5392 [consulta $10 / 04 / 2020]$.

Ley 1/2006. de Capitalidad y por la que se regula el Régimen Especial del municipio de Barcelona. Boletín Oficial del Estado, $\mathrm{n}^{\circ}$ 159, 2006, 13 de marzo, accesible en: https://www.boe.es/buscar/pdf/2006/BOE-A-2006-4583-consolidado.pdf [consulta 13/04/2020].

Ley 22/2006. de Capitalidad y de Régimen Especial de Madrid. Boletín Oficial del Estado, $\mathrm{n}^{\circ} 159$, 2006, 4 de julio, accesible en: https://www.boe.es/buscar/pdf/2006/BOE-A-2006-12057consolidado.pdf [consulta 11/04/2020].

Mallarach Isern, J. M., \& Vilagrasa i Ibarz, J. V. (2002) Los procesos de desconcentración urbana en las ciudades medias españolas. Ería: Revista cuatrimestral de geografía, (57), 57-70.

Martí Costa, M.; Barres González, R. y Rojas Rubio, M. (2018) "La metrópoli cercana. Gobernanza multinivel entre distritos e instituciones metropolitanas en Europa". Documento para su presentación en el IX Congreso Internacional en Gobierno, Administración y Políticas Públicas GIGAPP. (Madrid, España) del 24al 27de septiembre de 2018. Recuperado de https://cutt.ly/zhu1CaR [consulta 21/11/2020].

Martín Baeza, N. (2013) "Los presupuestos participativos: instrumento versus proceso participativo", en R Bañón i Martínez et al. (Coords.) Gestión de la escasez: participación, territorios y estado del bienestar: experiencias en democracia y participación. Madrid: Universidad Complutense de Madrid, pp. 202-210.

Montañés Serrano, M. (2013) "Metodologías participativas: justificación, fundamentación y descripción". En R Bañón i Martínez, R. Tamboleo García y G. Sánchez Medero (Coords.). Participación, democracia y gestión de la escasez: experiencias de democracia y participación. Madrid: Universidad Complutense de Madrid, pp. 315-319.

Ortiz Díaz, J., (1972) La desconcentración territorial en la administración local. Madrid: Instituto de Estudios de Administración Local.

Pindado, F, (2000) La participación ciudadana en las ciudades. Barcelona: Ediciones del Serbal.

Pineda Nebot, C. y Pires, V., (2014). "Estudio bibliométrico del Presupuesto Participativo en España", en R Bañón i Martínez, et al., (Coords.) La modernización de la política y la innovación participativa Madrid: Universidad Complutense de Madrid, pp. 19-36.

Prieto Romero, C., (2007) "Los Distritos en Madrid: proyecto de gestión desconcentrada" en Prieto Romero, Cayetano y Galán Galán, Alfredo, directores, Los distritos: gobierno de proximidad. Madrid: Thomson Civitas.

Prieto Romero, C. y Galán Galán, A. (dirs.), (2009) La descentralización de competencias autonómicas en la ciudad de Madrid. Nuevas oportunidades para la gestión desconcentrada. Madrid: Huygens Editorial.

Redacción (2010) "El fracaso de la consulta de la Diagonal se lleva por delante al Primer Teniente de Alcalde" en $L a$ Vanguardia, en https://www.lavanguardia.com/vida/20100516/53928946995/el-fracaso-de-la-consulta-de-ladiagonal-se-lleva-por-delante-al-primer-teniente-de-alcalde.html [consulta 15/05/2020].

R. O. 2004122, de Participación Ciudadana del Ayuntamiento de Madrid. Boletín Oficial del Ayuntamiento de Madrid, $\mathrm{n}^{\mathrm{o}}$ 5610, 2004, 29 de julio, accesible en: https://www.madrid.es/UnidadWeb/UGNormativas/Normativa/2004/Ficheros/ANM200422.pdf [consulta 23/04/2020].

R. O. 2004\23, del Gobierno y de la Administración del Ayuntamiento de Madrid. Boletín Oficial del Ayuntamiento de Madrid, $\mathrm{n}^{\mathrm{o}}$ 5610, 2004, 29 de julio, accesible en: https://www.madrid.es/UnidadWeb/UGNormativas/Normativa/2004/Ficheros/ANM23202004.pd f [consulta 20/04/2020].

R. O. 2005\8, de los Distritos de la Ciudad de Madrid. Boletín Oficial de la Comunidad de Madrid, $\mathrm{n}^{\circ}$
7 ,
2005 ,
10 de
enero,
accesible
en: 
https://www.madrid.es/UnidadesDescentralizadas/PerfilContratante/Contenidos/Fase1/Ficheros/ ANM2005_8.pdf [consulta 23/04/2020].

R. O. de Organización Municipal del Ayuntamiento de Barcelona. Boletín Oficial de la Provincia de Barcelona, $\mathrm{n}^{\circ}$ 64, 2001, 15 de marzo, accesible en: http://hdl.handle.net/11703/114343 [consulta 30/04/2020].

Reglamento de Participación Ciudadana del Ayuntamiento de Barcelona. Gaseta Municipal del Ayuntamiento de Barcelona, 2018, 8 de mayo, accesible en: http://hdl.handle.net/11703/109131 [consulta 02/05/2020].

Ruano de la Fuente, J. M. (2010) "Contra la participación: discurso y realidad de las experiencias de participación ciudadana", Política y Sociedad 47: 93-108.

Valero López, D. E. (2013) "Gobernanza local y participación ciudadana: la deliberación pública en el área metropolitana de Valencia”, en R Bañón i Martínez, R. Tamboleo García y G. Sánchez Medero (Coords.) Participación, democracia y gestión de la escasez: experiencias de democracia y participación. Madrid: Universidad Complutense de Madrid, pp. 202-210.

Vallès, J. M. y Brugué, Q., (2001) "El gobierno local” en M. Alcántara y A. Martínez (Coords.), Política y gobierno en España. Valencia: Tirant lo Blanch.

\section{Breve currículo}

\section{Rubén Tamboleo García}

Consultor Formación y Asuntos Públicos. Licenciado Ciencias Políticas y de la Administración, Licenciado Sociología, Máster Gobierno Administración Pública UCM. Autor artículos indexados, libros y capítulos diferentes editoriales. Director monografías Administración Pública. Profesor Visitante Campus Mahdía (Túnez). Investigador Visitante Universidades Liverpool y Viena. Investigador Proyectos Innovación Educativa, I+D+I empresas e Investigación Internacional COST-IS1308 COST-CA17132 COST-CA17135 y Horizon2020-“REMINDER”. Secretario Comité Científico Congresos Internacionales "Gobernanza y Asuntos Públicos" y "Democracias Populistas". Profesor y Director Programas Comunicación Política y Administración Pública. Secretario Académico "XII Aula Ortega Gasset" UIMP. Ponente 57 Congresos Internacionales. Evaluador ACAP, ECREA y COST. Analista político (laSexta, RT, Telemadrid, ABC,...). www.tamboleo.es

\section{Agradecimientos:}

Al Grupo de Investigación Gobernanza y Gestión Pública (GOGEP) de la Universidad Complutense de Madrid, que con el apoyo de Santander Universidades constituyó el marco idóneo para el desarrollo y la reflexión de investigaciones como las que ocupa este artículo. 